\title{
Estado nutricional e associação do excesso de peso com gênero e idade de crianças e adolescentes
}

\author{
Nutritional status and association of overweight with gender \\ and age in children and adolescents
}

\author{
Kleverton Krinski \\ Hassan Mohamed Elsangedy \\ Sergio da Hora \\ Cassiano Ricardo Rech ${ }^{2,3}$ \\ Elto Legnani \\ Bruno Vinicius Santos \\ Wagner de Campos \\ Sergio Gregorio da Silva
}

1 Universidade Federal do Paraná, Curitiba, PR, Brasil.

2 Universidade Estadual de Ponta Grossa, Ponta Grossa, PR. Brasil.

3 Bolsista Fundação Araucária.

Recebido em 05/03/10 Revisado em 20/08/10 Aprovado em 25/10/10
Resumo - O objetivo do presente estudo foi analisar o estado nutricional e verificar a associação do excesso de peso corporal (EPC) com o gênero e a idade em crianças e adolescentes da cidade de Vilhena, Rondônia. Participaram 5883 escolares, sendo crianças (6 a 11 anos) e adolescentes (12 a 17 anos), da rede pública de ensino. Foram mensuradas a massa corporal e estatura, e calculado o índice de massa corporal (IMC) para posterior classificação do estado nutricional. Utilizou-se o teste " $t$ " para comparação entre os gêneros e o teste do qui-quadrado e regressão de Poisson para verificar a associação do EPC com o gênero e a idade. A análise dos dados foi realizada no software SPSS, versão 11.0 e foi adotado nível de significância de p<0,05. A prevalência de EPC foi de 19,3\% nas crianças e 17,4\% nos adolescentes. Observou-se associação significativa entre EPC com o gênero e a idade entre as crianças, sendo que as meninas apresentaram maior prevalência de $\mathrm{EPC}(\mathrm{RP}=1,81$; $\left.\mathrm{IC}_{95 \%}=1,49-2,21\right)$ do que os meninos. Entre os adolescentes também se verificou que meninas apresentam maiores prevalência de $\mathrm{EPC}$ do que os meninos ( $\left.\mathrm{RP}=1,81 ; \mathrm{IC}_{95 \%}=1,49-2,21\right)$. A prevalência de EPC aumentou linearmente entre as crianças (6-11 anos) e diminuiu nos adolescentes (15-17 anos). Esses resultados sugerem que entre crianças e adolescentes do norte brasileiro as prevalências de excesso de peso apresentam-se elevadas.

Palavras-chave: Obesidade; Sobrepeso; Criança; Adolescência.

Abstract - The aim of this study was to assess the nutritional status and the association of excess body weight (EBW) with gender and age among children and adolescents from the town of Vilhena, Rondonia, Brazil. A total of 5,883 students, including children (6 to 11 years) and adolescents (12 to 17 years), from public schools participated in the study. Body weight and height were measured and the body mass index was calculated for subsequent classification of nutritional status. The t-test was used for comparison between genders and the chi-square test and Poisson regression were used to evaluate the association between EBW, gender, and age. Data were analyzed with the SPSS 11.0 program at a level of significance of $p<0.05$. The prevalence of EBW was $19.3 \%$ among children and $17.4 \%$ among adolescents. EBW was significantly associated with gender and age among children. In addition, the prevalence of $E B W$ was higher among girls $(P R=1.81$, 95\% CI 1.49 to 2.21) than among boys. Adolescent girls also presented a higher prevalence of $E B W$ than boys ( $P R=1.81,95 \%$ CI 1.49 to 2.21). The prevalence of EBW increased linearly in children (6 to 11 years) and decreased in adolescents (15 to 17 years). These results suggest that the prevalence of overweight is high among children and adolescents from northern Brazil. Key words: Obesity; Overweight; Child; Adolescence 


\section{INTRODUÇÃO}

A literatura científica tem documentado uma transição nutricional no Brasil nos últimos anos ${ }^{1}$. Em menos de duas décadas passou-se de um quadro de desnutrição grave para uma epidemia de obesida$\mathrm{de}^{2,3}$. Esta transição é atribuída a inúmeros fatores como a urbanização, melhora nas condições de vida da população, mudanças no estilo de vida relacionada ao aumento da prevalência do sedentarismo, e maior consumo de alimentos hipercalóricos ${ }^{3}$.

Paralelamente a esta elevada prevalência de excesso de peso corporal (EPC), nota-se concomitantemente um aumento das morbidades associadas a este distúrbio, como por exemplo, a diabetes, hipertensão, hipercolesterolêmica, doenças cardiovasculares e desenvolvimento da síndrome metabólica ${ }^{4,5}$. Em crianças e adolescentes os efeitos adversos da obesidade também são bem documentados, e direcionam para um quadro preocupante, devido ser neste período que diversos comportamentos são adquiridos ${ }^{4,6}$.

No Brasil, de acordo com revisão sistemática que trata do tema da obesidade em adolescentes ${ }^{7}$, verifica-se que a maior parte dos estudos realizados com adolescentes é proveniente de amostras das regiões sul e sudeste, com poucos estudos nas regiões norte e nordeste, sendo a maioria com levantamentos transversais e amostras locais? ${ }^{7}$. Exceção se faz ao estudo realizado na cidade de Pelotas ${ }^{8}$, que utilizou delineamento de coorte.

Estudos com amostras regionais do norte do Brasil são escassos?. Dois levantamentos com crianças e adolescentes ${ }^{9,10}$, com idades entre 7 a 15 anos, da cidade de Porto Velho-RO, demonstraram elevada prevalência de casos de adiposidade corporal em excesso (23,7\%), desnutrição pregressa $(27,2 \%)$ e desnutrição aguda (19,8\%).

Entende-se que existem diferenças consideráveis no desenvolvimento tecnológico e econômico entre as regiões brasileiras, e até mesmo dentro de uma mesma região. Deste modo, a realidade da transição nutricional observada no sul e sudeste do país parece ter diferentes magnitudes em outras regiões. Além disto, a relação da obesidade com gênero e idade estabelecida em estudos nas regiões sul e sudeste, não foi verificada em amostras de crianças e adolescentes da região norte do Brasil.

Assim, o presente estudo teve como objetivo: identificar o estado nutricional em crianças e adolescentes da cidade de Vilhena, Rondônia, assim como, verificar a associação entre o excesso de peso corporal (sobrepeso/obesidade) com o gênero e a idade.

\section{PROCEDIMENTOS METODOLÓGICOS}

\section{Delineamento do estudo}

Realizou-se um estudo descritivo, transversal de base populacional escolar, com crianças e adolescentes entre seis (6) e dezessete (17) anos de idade, de ambos os sexos, matriculados na rede pública estadual de ensino do Município de Vilhena, Rondônia.

O presente estudo foi aprovado pelo Comitê de Ética em Pesquisa da Universidade Federal do Paraná (Parecer 332/2006) e acompanhou as normas da Resolução 196/96 do Conselho Nacional de Saúde sobre pesquisa envolvendo seres humanos. Além disso, todos os alunos envolvidos na pesquisa e seus respectivos responsáveis assinaram voluntariamente um termo de consentimento livre e esclarecido, no qual foram informados quanto aos objetivos do estudo e esclarecidos quanto aos métodos utilizados, ficando garantido aos participantes o anonimato e o direito de desistir do estudo a qualquer momento.

\section{População e Amostra}

A população de escolares foi estimada por meio do censo escolar de 2007, o qual divulgou que, no município de Vilhena-RO, haviam, aproximadamente, 15942 alunos, regularmente matriculados na rede pública de ensino. Desses, um total de aproximadamente 7875 alunos, distribuídos em quatro (4) escolas da rede pública estadual, sendo 2315 de $1^{\circ}$ a $4^{\circ}$ série, 3109 de $5^{\circ}$ a $8^{\circ}$ série e 2451 do Ensino Médio. Foram selecionadas para o estudo todas as escolas da rede Estadual de Ensino.

O tamanho da amostra foi determinado a partir do cálculo amostral ${ }^{11}$, considerando uma prevalência de EPC estimada em $20 \%{ }^{12}$, um cálculo de erro amostral de 3\% e efeito do desenho do estudo de 2,013. Ainda foi acrescido na amostra final um percentual destinado a possíveis perdas ou erros de medida (20\%). Deste modo a amostra mínima foi estimada em 1508 escolares, considerando a proporcionalidade em relação ao agrupamento da série de estudo $\left(1^{\circ}\right.$ a $4^{\circ}$ série, $5^{\circ}$ a $8^{\circ}$ série e ensino médio). Devido às condições logísticas de aplicação do estudo e delineamento metodológico que incluiu poucas variáveis na coleta de dados, foi possível incluir na amostra 5883 escolares, sendo 2765 crianças (6 a 11 anos) e 3118 adolescentes (12 a 17 anos). Adotou-se como critério de exclusão: adolescente que se recusaram em participar do estudo, idade $\geq 18$ anos, gravidez ou indivíduos que apresentassem alguma necessidade especial.

\section{Procedimentos}

A coleta de dados ocorreu durante o primeiro se- 
mestre do ano de 2008, durante o horário destinado à disciplina de Educação Física. Inicialmente, os pesquisadores entregaram o termo de consentimento livre e esclarecido para a autorização do responsável e do próprio aluno para participação no estudo. No dia seguinte, os escolares que voluntariamente desejassem participar do estudo tiveram suas medidas de massa corporal e estatura mensuradas?

Forma utilizados um estadiômetro de alumínio com resolução de $1 \mathrm{~cm}$ e duas balanças digitais da marca Plenna com resolução de 100 gramas, aferida a cada dez pesagens, com intuito de manter a precisão da medida. As medidas foram tomadas por dois avaliadores treinados e com erro técnico de medida inferior a 0,5\% para as medidas de massa corporal e estatura. A partir das medidas antropométricas foi calculado o índice de massa corporal (IMC) e classificado em baixo peso, normoponderal e excesso de peso (sobrepeso + obeso) $)^{13}$. A utilização deste ponto de corte se deve, em virtude de estudos prévios ${ }^{14,15}$, reportarem que entre as referências nacionais e internacionais, este critério apresentou uma maior sensibilidade entre crianças e adolescentes brasileiros.

\section{Analise Estatística}

Para o tratamento estatístico, utilizou-se, inicialmente, a estatística descritiva (frequência, média e desvio-padrão). O teste "t" de Student para amostras independentes foi utilizado para comparação de médias entre os gêneros. Para verificar a associação entre os casos de excesso de peso (sobrepeso/ obesidade) e as variáveis de gênero e idade foi utilizado o teste de associação do qui-quadrado $\left(\chi^{2}\right)$ e calculada a razão de prevalência (RP) com seu respectivo intervalo de confiança (IC95\%), por meio da regressão de Poisson. A análise dos dados foi realizada no software SPSS, versão 11.0 e foi adotado nível de significância de p<0,05.

\section{RESULTADOS}

Foram avaliados 5883 escolares, sendo 2765 crianças (6 a 11 anos) e 3118 adolescentes (12 a 17 anos). A tabela 1 apresenta as características descritivas das crianças e adolescentes participantes do estudo. Observa-se que entre as crianças não houve diferenças estatísticas $(p>0,05)$ entre os gêneros, já no grupo dos adolescentes foram encontradas diferenças estatísticas $(\mathrm{p}<0,05)$ para todas as variáveis físicas analisadas.

Com relação ao estado nutricional (Figura 1), baseado nas medidas de IMC, a prevalência de EPC foi de 19,3\% ( IC $\left._{95 \%}: 16,3-24,2 \%\right)$, nas crianças e $17,4 \%\left(\mathrm{IC}_{95 \%}: 14,5-22,9 \%\right)$ entre os adolescentes. Entre as crianças, as meninas apresentaram prevalência de baixo peso $\left(5,2 \%, \mathrm{IC}_{95 \%}: 3,1-7,3 \%\right)$ e excesso de peso $\left(19,7 \%, \mathrm{IC}_{95 \%}: 16,6-25,2 \%\right)$ mais elevada do que nos meninos $\left(2 \%, \mathrm{IC}_{95 \%}: 1,1-3,5 \%\right.$; $\left.17,9 \%, \mathrm{IC}_{95 \%}: 14,3-28,2 \%\right)$, respectivamente, demonstrando associação estatística do estado nutricional com o gênero ( $\mathrm{p}<0,01)$. Nos adolescentes, também houve a mesma tendência. As meninas apresentaram maiores prevalências de baixo peso $\left(4,7 \% . \mathrm{IC}_{95 \%}: 2,4-6,5 \%\right)$ e excesso de peso $(14,6 \%$. $\left.\mathrm{IC}_{95 \%}: 13,3-18,9 \%\right)$, quando comparadas com os meninos $(\mathrm{p}<0,01)$

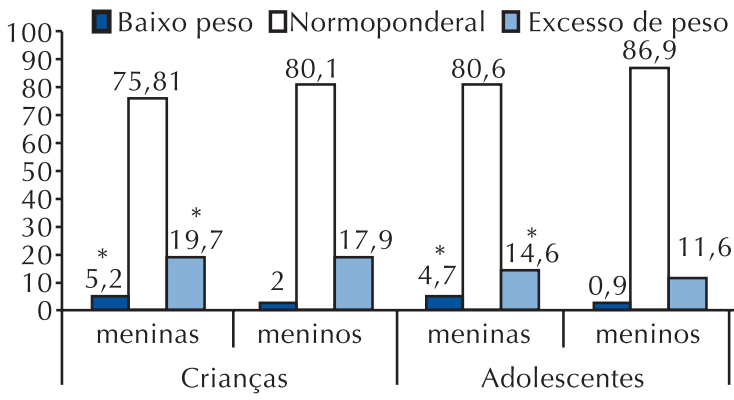

Figura 1. Prevalência de baixo peso, normoponderal e excesso de peso em crianças e adolescentes do Município de Vilhena, Rondônia, 2008, de acordo com o gênero.

* Associação (teste qui-quadrado) do gênero com o estado nutricional de crianças e adolescentes $(p<0,01)$.

Tabela 1. Valores de média e desvio-padrão das características físicas de crianças e adolescentes do Município de Vilhena, Rondônia, 2008.

\begin{tabular}{lcccc}
\hline \multirow{2}{*}{ Variáveis } & \multicolumn{2}{c}{ Crianças } & \multicolumn{2}{c}{ Adolescentes } \\
\cline { 2 - 5 } & $\begin{array}{c}\text { Meninos } \\
(\mathrm{n}=1.370)\end{array}$ & Meninas $(\mathrm{n}=1.395)$ & Meninos $(\mathrm{n}=1.393)$ & Meninas $(\mathrm{n}=1.725)$ \\
\hline Idade (anos) & $8,8 \pm 1,6$ & $8,8 \pm 1,6$ & $13,9 \pm 1,5$ & $14,2 \pm 1,5 \dagger$ \\
Peso $(\mathrm{kg})$ & $30,6 \pm 8,7$ & $30,9 \pm 9,5$ & $52,2 \pm 12,5$ & $50,3 \pm 10,3 \dagger$ \\
Estatura $(\mathrm{cm})$ & $134 \pm 1,07$ & $135 \pm 1,22$ & $163 \pm 1,11$ & $158 \pm 0,68 \dagger$ \\
IMC $\left(\mathrm{kg} / \mathrm{m}^{2}\right)$ & $16,6 \pm 2,6$ & $16,8 \pm 3,3$ & $19,2 \pm 3,2$ & $19,8 \pm 3,3+$ \\
\hline
\end{tabular}

IMC: Índice de massa corporal.

* difere estatisticamente $\mathrm{p}<0,05$ entre os gêneros dentro do grupo (teste $t$ independente). 
Observou-se uma tendência linear de aumento da prevalência de EPC de acordo com a idade nas crianças. Houve um aumento significativo de 18 pontos percentuais de EPC entre as idades de seis e 11 anos. Por outro lado, nos adolescentes, notou-se uma relação inversa nesta associação (figura 2). Houve uma diminuição estatística de cerca de 10 pontos percentuais dos valores de prevalência de excesso de peso entre 12 e 16 anos de idade.

A prevalência de EPC também foi analisada em relação aos ajustes para gênero e idade. As tabelas 2 e 3 apresentam os valores de razão de prevalência e seus respectivos intervalos de confiança $\left(\mathrm{IC}_{95 \%}\right)$ para crianças e adolescentes no presente estudo. A maior prevalência de EPC, entre as crianças (tabela 2), foi observada entre as meninas ( $\mathrm{RP}=1,81$; IC $95 \%=1,49$ 2,21) quando comparadas com os meninos. Estes valores são estatisticamente significativos mesmo quando ajustados para a idade. Os resultados demonstram que uma criança com 11 anos possui 2,55 maior prevalência de EPC quando comparada com uma criança de seis (6) anos de idade.

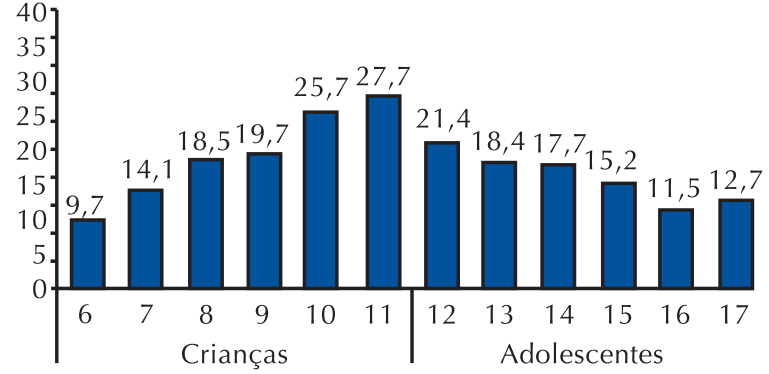

Figura 2. Prevalência e associação dos escores de excesso de peso e idade em crianças e adolescentes do Município de Vilhena, Rondônia.

Associação (teste qui-quadrado para tendência) $p<0,01$.

No grupo dos adolescentes, as diferenças entre os gêneros foram menores do que aquelas apresentadas nas crianças. Porém a prevalência de EPC esteve associada ao gênero feminino e a idade, somente entre as idades de 15 e 17 anos. Contudo, a associação com a idade foi inversamente proporcional, demonstrando que a idade pode ser vista como um fator de proteção entre os adolescentes para o desenvolvimento do EPC.

Tabela 2. Valores de razão de prevalência entre excesso de peso e idade em crianças do Município de Vilhena, Rondônia, 2008.

\begin{tabular}{|c|c|c|c|c|c|}
\hline \multirow{2}{*}{ Variáveis } & \multirow{2}{*}{$\%$} & \multicolumn{2}{|c|}{ Análise Bruta } & \multicolumn{2}{|c|}{ Análise Ajustada** } \\
\hline & & RP & $\mathrm{IC}_{95 \%}$ & RP & $\mathrm{IC}_{95 \%}$ \\
\hline \multicolumn{6}{|l|}{ Sexo } \\
\hline Masculino $(n=1.370)$ & 14,7 & 1 & - & 1 & - \\
\hline Feminino $(n=1.395)$ & 23,7 & $1,79^{*}$ & $1,48-2,18$ & $1,81^{*}$ & $1,49-2,21$ \\
\hline \multicolumn{6}{|l|}{ Idade (anos) } \\
\hline $6(n=288)$ & 9,7 & 1 & - & 1 & - \\
\hline $7(n=398)$ & 14,1 & $1,52^{*}$ & $1,01-2,46$ & $1,59 *$ & $1,03-2,59$ \\
\hline $8(n=466)$ & 18,5 & $2,10^{*}$ & $1,33-3,31$ & $2,16^{*}$ & $1,37-3,42$ \\
\hline $9(n=487)$ & 19,7 & $2,28^{*}$ & $1,45-3.57$ & $2,38^{*}$ & $1,51-3,73$ \\
\hline $10(n=535)$ & 25,7 & $3,55^{*}$ & $2,30-5,47$ & $2,36^{*}$ & $2,39-5,72$ \\
\hline $11(n=591)$ & 27,7 & $2,34^{*}$ & $1,51-3,63$ & $2,55^{*}$ & $1,64-3,98$ \\
\hline
\end{tabular}

$\mathrm{RP}=$ razão de prevalência. $\mathrm{IC}_{95 \%=}$ intervalo de confiança $95 \%$. * associação significativa $\mathrm{p}<0,001$. ** Análise ajustada para sexo e idade.

Tabela 3. Valores de associação entre excesso de peso e idade em adolescentes do Município de Vilhena, Rondônia, 2008.

\begin{tabular}{|c|c|c|c|c|c|}
\hline \multirow{2}{*}{ Variáveis } & \multirow{2}{*}{$\%$} & \multicolumn{2}{|c|}{ Análise Bruta } & \multicolumn{2}{|c|}{ Análise Ajustada** } \\
\hline & & $\mathrm{RP}$ & $\mathrm{IC}(95 \%)$ & $\mathrm{RP}$ & IC (95\%) \\
\hline \multicolumn{6}{|l|}{ Sexo } \\
\hline Masculino $(n=1.393)$ & 11,6 & 1 & & 1 & \\
\hline Feminino $(n=1.725)$ & 14,6 & 1,02 & $0,87-1,19$ & $1,08^{*}$ & $1,00-1,21$ \\
\hline \multicolumn{6}{|l|}{ Idade (anos) } \\
\hline $12(n=618)$ & 21,4 & 1 & & 1 & \\
\hline $13(n=631)$ & 18,4 & 0,83 & $0,63-1,08$ & 0,82 & $0,62-1,09$ \\
\hline $14(n=651)$ & 17,7 & 0,79 & $0,58-1,03$ & 0,78 & $0,59-1,04$ \\
\hline $15(n=593)$ & 15,2 & 0,66 & $0,49-0,88$ & $0,65^{*}$ & $0,49-0,88$ \\
\hline $16(n=419)$ & 11,5 & 0,47 & $0,33-0,69$ & $0,47^{*}$ & $0,33-0,68$ \\
\hline $17(n=205)$ & 12,7 & 0,52 & $0,34-0,84$ & $0,53^{*}$ & $0,34-0,84$ \\
\hline
\end{tabular}

$\mathrm{RP}=$ razão de prevalência. IC95\% = intervalo de confiança 95\%. * associação significativa p<0,001.** Análise ajustada para sexo e idade. 


\section{DISCUSSÃO}

O aumento da epidemia de obesidade tem sido observado em diversos países ${ }^{3}$. Da mesma forma, nota-se que este aumento tem atingido todas as faixas etárias e níveis socioeconômicos ${ }^{16,17}$. O presente estudo procurou investigar a realidade do estado nutricional e da associação do EPC com as variáveis de gênero e idade entre escolares (crianças e adolescentes) de uma cidade da região norte do Brasil. Alguns pontos metodológicos merecem destaque nas análises e repercussão dos resultados e conclusões aqui apresentadas. A amostra, apesar de ser representativa e com poder estatístico para as análises empregadas, foi obtida com escolares da rede estadual de ensino, assim, os resultados devem ser vistos com cautela para a extrapolação para outros grupos de adolescentes como: os não escolares, alunos de escolas particulares e grupos indígenas que vivem na região do estudo. Outra limitação relaciona-se à utilização do IMC, que por natureza matemática e lógica, não mede a adiposidade corporal, contudo, é aceito como uma medida internacional para a classificação populacional de excesso de peso e está associado com riscos à saúde ${ }^{16-19}$.

As análises foram realizadas com a estratificação da amostra em dois grupos: crianças e adolescentes; pois as diferenças atribuídas ao desenvolvimento maturacional são consideráveis entre as idades ${ }^{20}$. O fato de os adolescentes desse estudo não terem sido classificados de acordo com os níveis de maturação biológica, também pode ter contribuído para a maior associação entre EPC e a idade. Mesmo considerando estas limitações, outros estudos foram realizados com o mesmo delineamento e são importantes para fins de diagnóstico e controle epidemiológico da realidade e mudanças em um dado espaço de tempo ${ }^{1,9}$.

Os resultados do presente estudo permitem verificar e confirmar a teoria de que as crianças e adolescentes de cidades do norte do Brasil, também passam por um processo de transição nutricional importante. No norte do Brasil, observou-se que houve uma redução de 39\% para 23\% da desnutrição em crianças e adolescentes nas últimas décadas ${ }^{17}$. Estes valores são próximos aos relatados em crianças $^{9}$ e adolescentes ${ }^{12}$ de Porto Velho-RO. No presente estudo, as prevalências de baixo peso entre as crianças foram menores do que as apresentada por Farias et al. ${ }^{9}$, isso pode ter sido decorrente das diferenças verificadas na amostra estudada, a qual foi constituída somente por crianças de baixo nível socioeconômico, fator este que não foi avaliado na presente investigação, sendo que, desta forma, poderiam estar inseridos na amostra crianças e adolescentes com diferentes status socioeconômicos. Em relação aos adolescentes, foi verificado uma menor prevalência de baixo peso, quando comparado a outro estudo realizado por FARIAS et al. ${ }^{21}$. Isso pode ter ocorrido devido à utilização de diferentes indicadores antropométricos e pontos de corte para o diagnóstico dos casos de desnutrição.

A prevalência de EPC entre as crianças (19,3\%) e adolescentes (17,4\%) foi similar aos resultados apresentados em adolescentes Europeus ${ }^{22}$ e superior aos relatados nas regiões nordeste e sudeste do Brasil ${ }^{16}$. Porém foi concordante com os achados no Suñé et al., ${ }^{19}$, os quais avaliaram um grupo de escolares de 11 a 13 anos de idade, reportando uma elevada taxa de sobrepeso e obesidade em torno de $24,8 \%$. De maneira semelhante Terres et al., ${ }^{23}$ após avaliarem uma amostra de adolescentes residentes na cidade de Pelotas-RS, com idades entre 15 e 18 anos, registraram uma prevalência de 20,9\% de sobrepeso.

Prévios estudos ${ }^{19,24,25}$ têm demonstrado que este aumento na prevalência de sobrepeso e obesidade entre crianças e adolescentes parece estar relacionado a questões vinculadas a modificação do estilo de vida, levando as crianças e adolescentes a passar mais tempo em atividades sedentárias, o que pode implicar um rápido aumento da epidemia de obesidade no Brasil ${ }^{25}$

Da mesma forma, grande parte dos estudos realizados nas regiões Sul, Sudeste e Nordeste do Brasil ${ }^{16,19,26}$, demonstram que o EPC está associado ao gênero, com meninas apresentando maiores prevalências quando comparadas com os meninos, tanto em crianças quanto em adolescentes. Estes resultados são semelhantes aos relatados por Guedes et al..$^{27} \mathrm{em}$ um recente estudo conduzido na cidade de Montes Claros-MG, os quais analisaram 2849 escolares de 6 a 18 anos, revelando que, entre as crianças, as meninas demonstram uma maior chance para desenvolver EPC, comparado aos meninos e um aumento linear para o desenvolvimento deste quadro com o aumento da idade em ambos os gêneros.

De acordo com Malina e Bouchard ${ }^{20}$, o engajamento dos meninos em atividades de maior gasto calórico, como atividades vigorosas (correr, saltar, jogos de bola), podem explicar esse quadro, isto porque, estes apresentam menor prevalência de EPC. Entre as meninas adolescentes, também parece haver uma maturação sexual antecipada quando comparada com a idade cronológica dos meninos, 
assim, as meninas possuem uma prevalência maior de excesso de peso $^{20}$.

A associação entre o EPC e a idade foi significativa $(p<0,01)$, tanto nas crianças quanto nos adolescentes, contudo, o sentido da associação foi diferente. Nas crianças, houve um aumento de $9,7 \%$ aos seis anos para $27,7 \%$ aos 11 anos, ou seja, houve um aumento linear da prevalência de EPC com a idade. Deve-se observar que, na infância, o aumento de peso é mais acentuado que os ganhos em estatura, isso talvez por que estas crianças não tenham atingido o ápice maturacional ${ }^{20}$.

Já entre os adolescentes houve uma associação inversa. A idade se mostrou como um fator protetor para o excesso de peso. A preocupação com a imagem corporal, a adoção de maiores níveis de atividade física (de lazer e principalmente de trabalho) e um maior controle do consumo alimentar, podem ser fatores que estejam contribuindo para esta associação inversa. Além disso, é fato que, nas regiões norte e nordeste, os adolescentes contribuam com a sua força de trabalho para o aumento da renda da família, isso pode promover impactos significativos no aumento do nível de atividades físicas, aumentando o gasto calórico e assim diminuir a prevalência de $\mathrm{EPC}^{28}$.

Com isso, a partir dos resultados desse estudo pode-se concluir que são elevadas as prevalências de EPC, entre escolares (crianças e adolescentes) da região Norte do Brasil. Também se observou que o gênero e a idade estão associados ao EPC de peso de forma independente, tanto nas crianças quanto nos adolescentes. Assim, sugere-se que futuros estudos sejam realizados a fim de diagnosticar os padrões e as preferências de atividades físicas em relação ao gênero e a idade, com o intuito de propor intervenções mais eficientes nesta região do Brasil e assim auxiliar no combate a epidemia da obesidade.

\section{REFERÊNCIAS BIBLIOGRÁFICAS}

1. Chaves VLV, Freese E, Lapa MT, Cesse EAP, Vasconcelos ALR. Evolução espaço-temporal do sobrepeso e da obesidade em adolescentes masculinos brasileiros, 1980 a 2005. Cad Saúde Pública 2010;26(7):1303-13.

2. Coutinho JG, Gentil CP, Toral N. A desnutrição e obesidade no Brasil: o enfrentamento com base na agenda única da nutrição. Cad Saúde Pública 2008;24(Sup. 2):332-40.

3. World Health Organization/WHO. Obesity: preventing and managing the global epidemic. Geneva: World Health Organization. 2000; Available from: <http:// www.who.int/whr/2000/en/> [2008 May 20).
4. Rolland-Cachera MF, Deheeger M, Maillot M, Bellisle F. Early adiposity rebound: causes and consequences for obesity in children and adults. Int J Obes 2006;30(Sup. 4):11-7.

5. Juárez-López C, Klünder-Klünder M, Medina-Bravo P, Madrigal-Azcárate A, Mass-Díaz E, Flores-Huerta S. Insulin resistance and its association with the components of the metabolic syndrome among obese children and adolescents. BMC Public Health 2010;7(10):318-25.

6. Moraes ACF, Fulaz CS, Netto-Oliveira ER, Reichert FF. Prevalência de síndrome metabólica em adolescentes: uma revisão sistemática. Cad Saúde Pública 2009;25(6):1195-202.

7. Hallal PC, Wells JCK, Reichert FF, Anselmi L, Victora CG. Early determinants of physical activity in adolescence: prospective birth cohort study. BMJ 2006;332(7548):1002-5.

8. Victora CG, Barros F, Lima RC, Horta BL, Wells J. Anthropometric and body composition of 18 year old men according to duration of breast feeding: birth cohort study from Brazil. BMJ 2003;327(7420):901-6.

9. Farias ES, Guerra-Junior G, Petroski ED. Estado nutricional de escolares em Porto Velho, Rondônia. Rev Nutr 2008;21(4):401-9.

10. Farias ED, Petroski EL. Estado nutricional e atividade física de escolares da cidade de Porto Velho, RO. Rev Bras Cineantropom Desempenho Hum 2003;5(1):27-38.

11. Magnanini MMF, Luiz RR. A lógica da determinação do tamanho da amostra em investigações epidemiológicas. Cad Saúde Colet 2000;8(2):9-28.

12. Tassitano RM, Bezerra J, Tenório MCM, Colares V, Barros MVG Hallal PC. Physical activity in brasilian adolescents. A systematic review. Rev Bras Cineantropom Desempenho Hum 2007;9(1):55-60.

13. Conde WL, Monteiro CA. Valores críticos do índice de massa corporal para classificação do estado nutricional de crianças e adolescentes brasileiros. J Pediatr 2006;82(4):266-72.

14. Vitolo MR, Campagnolo PDB, Barros ME,Gama CM,Lopez FA. Evaluation of two classifi cátions for overweight among Brazilian adolescents. Rev Saúde Publica 2007;41(4):653-6.

15. Glaner MF. Índice de massa corporal como indicativo da gordura corporal comparado às dobras cutâneas. Rev Bras Med Esporte 2005;11(4):243-6.

16. Wang Y, Monteiro C, Popkin BM. Trends of obesity and underweight in older children and adolescents in the United States, Brazil, China and Rússia. Am J Clin Nutr 2002;75(6):971-7.

17. Campos LA, Leite AJ, Almeida PC. Nível socioeconômico e sua influência sobre a prevalência de sobrepeso e obesidade em escolares adolescentes do município de Fortaleza. Rev Nutr 2006;19(5):531-8.

18. Reilly JJ, Methven E, McDowell ZC, Hacking B, Alexander D, Stewart D, et al. Health consequences of obesity. Arch Dis Child 2003;88(9):748-52. 
19. Balban G, Silva GAP, Motta MEFA. Prevalência de sobrepeso e obesidade em escolares de diferentes classes socioeconômicas em Recife, PE. Pediatria 2001;23(4):285-9.

20. Malina R, Bouchard C. Atividade física do atleta jovem: do crescimento a maturação. São Paulo: Roca; 2002.

21. Farias ES, Salvador MRD. Antropometria, composição corporal e atividade física em escolares. Rev Bras Cineantropom Desempenho Hum 2005;7(1):21-9.

22. Ekelund U, Brage S, Froberg K, Harro M, Anderssen AS, Sardinha LB. TV viewing and physical activity are independently associated with metabolic risk in children: The European Youth Heart Study. Plos Med 2006;3(12):2449-57.

23. Terres NG, Pinheiro RT, Horta BL, Pinheiros KAT, Hotra LL. Prevalence of factors associated to overweight and obesity in adolescents. Rev Saúde Publica 2006;40(4):1-6.

24. Monteiro P, Victora C, Barros F. Fatores de risco sociais, familiares e comportamentais para a obesidade em adolescentes. Rev Panam Salud Publ 2004;16(4):250-7.

25. Suñé FR, Dias-da-Costa JS, Olinto MTA, Pattussi MP. Prevalência e fatores associados para sobrepeso e obesidade em adolescentes de uma cidade no Sul do Brasil. Cad Saúde Publica 2007;23(6):1361-71.
26. Soar C, de Vasconcelos FAG, de Assis MAA e Grosseman S. Prevalência de sobrepeso e obesidade de uma escola pública de Florianópolis, Santa Catarina. Rev Bras Saúde Matern Infant 2004;4(4):391-7.

27. Guedes DP, Neto JTM, Almeida MJ, Silva AJRM. Impacto de fatores sociodemográficos e comportamentais na prevalência de sobrepeso e obesidade de escolares. Rev Bras Cineantropom Desempenho Hum 2010;12(4):221-31.

28. Instituto Brasileiro de Geografia e Estatística (IBGE). Trabalho Infantil. 2001; Disponível em: http://www. ibge.gov.br/home/estatistica/populacao/condicaodevida /trabalhoinfantil/default.shtm> [2009 dez 05].

\section{Endereço para correspondência}

Sergio Gregorio da Silva

Rua Coração de Maria, 92 - JD Botânico

80.215-370 - Curitiba, PR - Brasil

E-mail: sergiogregorio@ufpr.br 